\title{
A new approach to investigate wave dissipation in viscoelastic tubes: application of Wave Intensity Analysis
}

\author{
J. Feng and A.W. Khir \\ School of Engineering and Design, Brunel University, Uxbridge, Middlesex, UK, UB8 3PH
}

\begin{abstract}
Wave dissipation in elastic and viscoelastic medium has been investigated extensively in the frequency domain. The aim of this study is to examine the pattern of wave dissipation in the time-domain using Wave Intensity Analysis. A single semi-sinusoidal pulse was generated in $8 \mathrm{~mm}$ and $16 \mathrm{~mm}$ diameter tubes; each is of $200 \mathrm{~cm}$ in length. Pressure and flow measurements were taken at intervals of $5 \mathrm{~cm}$ along the tube. In order to examine the effect of the wall mechanical properties on wave dissipation, we also modified the wall of the $16 \mathrm{~mm}$ tube; a thread of strong cotton was wound with a pitch of approximately $30^{\circ}$ around the circumference of the tube in the longitudinal direction. The separated forward pressure, wave intensity and wave energy were calculated using Wave Intensity Analysis. The amplitudes of the forward pressure wave, wave intensity and wave energy dissipated exponentially with distance. In the $8 \mathrm{~mm}$ diameter tube, the dissipation of forward pressure, wave intensity and wave energy were greater than those in $16 \mathrm{~mm}$ tube. For the same sized of tube, there was no significant difference in the dissipation of forward pressure, wave intensity and wave energy between the modified and normal wall tubes. It is concluded that the size of tube has a significant effect on the wave dissipation but the mechanical properties of the wall do not have a discernable effect on wave dissipation.
\end{abstract}

\section{INTRODUCTION}

$\mathrm{O}$ VER the past few decades wave propagation in blood vessels and viscoelastic tubes has been examined extensively. Anliker $[1,2,3,4]$ examined the wave transmission characteristic in blood vessels in the frequency domain and found that wave dissipated exponentially with the distance travelled. Anliker [5] concluded that the damping of the various waves in any vessels may be attributed to the following possible mechanisms: (1) radiation into the surrounding tissue, (2) dissipation due to the viscosity of the blood and (3) the viscoelasticity of the vessel wall. Theoretical results have shown the viscosity to be of minor importance to wave dissipation. Anliker [4] also demonstrated that the radiation of surrounding medium has little effect on wave dissipation. It thus appears that the primary mechanism for wave dissipation is the viscoelasticity of the wall itself. Newman [6] also examined the pulse wave propagation in viscoelastic medium in the frequency domain experimentally and concluded that pulse wave amplitude attenuated along the travelling distance as monoexponential reduction function in the latex tube. However, conclusions from Ursino [7] and Bertram [8] had shown considerable disparity in the estimations of wave dissipation values, and different explanations about the reasons for this disparity have been suggested.
This study explores wave dissipation in the time domain using Wave Intensity Analysis [9], which is a relatively new method that proved useful in studying waves in the arterial system. The method has the advantage of not assuming any periodicity or linearity in the system. The method is also a time-domain analysis, which will enable us to study wave dissipation both in time and space.

The measured waves are the summation of the forward and the backward (reflected) waves which makes it more complicated to examine wave dissipation along a certain direction. Wave Intensity Analysis simplifies this problem by separating the measured wave into their forward and backward directions, and waves dissipation is then examined using the forward pressure instead of the measured pressure.

Furthermore, Wave Intensity Analysis makes it possible to examine wave dissipation in terms of energy. Herein we present the experimental result about wave energy dissipation along the travelled distance.

\section{THEORETICAL BACKGROUND}

Parker and Jones [9] introduced a method, to separate the pressure and velocity waveforms into their forward and backward directions. The theoretical work is based on the solution of the one-dimension equations of conservation of mass and momentum in elastic tubes, which are

$$
\begin{aligned}
& A_{t}+(U A)_{x}=0 \\
& U_{t}+U U_{x}+\frac{P_{x}}{\rho}=0
\end{aligned}
$$

Where $P, U$ are pressure and velocity in the tube over the time $t$. $A$ is cross-section area of tube, $\rho$ is density and $x$ is the wave travelled distance. The water hammer equation is

$$
d P_{ \pm}= \pm \rho c d U_{ \pm}
$$

Where \pm denotes the forward and backward direction, $d P_{ \pm}$and $d U_{ \pm}$denote the forward and backward wavefronts of pressure and velocity, $c$ is wave speed. Wave intensity, $d I$, which is the energy carried by the wave per cross-section area [10], is defined as the product of $d P$ and $d U$, which can be expressed as:

$d I=d P d U$ 
Where, $d P$ and $d U$ denote changes in the pressure, $P$ and velocity $U$, respectively. The forward and backward pressure [12] can be calculated as

$$
\begin{aligned}
& d P_{ \pm}=\frac{1}{2}(d P \pm \rho c d U) \\
& P_{+}=P_{0}+\sum d P_{+}
\end{aligned}
$$

Where, $P_{0}$ and $P_{+}$are initial static pressure and forward pressure respectively.

Separated wave intensity [11] can be calculated as

$$
d I_{ \pm}= \pm \frac{1}{4 \rho c}(d P \pm \rho c d U)^{2}
$$

The wave intensity has a unit of $W / \mathrm{m}^{2}$ and its integration, which we call 'wave energy', will have units of ' $\mathrm{J} / \mathrm{m}^{2}$ '.

\section{METHOD}

\section{A. Experiment setup}

Wave dissipation was investigated experimentally with two sizes of latex tubes, $8 \mathrm{~mm}$ and $16 \mathrm{~mm}$ in diameter; each is of $200 \mathrm{~cm}$ in length (see Fig.1). A rectangular tank filled water, $50 \mathrm{~cm}$ in width and $200 \mathrm{~cm}$ in length, was used to support tubes. The inlet and outlet of the tank were connected with two small reservoirs, whose level can be adjusted to provide initial head. The level of water in the tank was just above the tube. This guaranteed a uniform transmural pressure and support of tube. A piston pump produced one forward semi-sinusoidal signal at the inlet of the tube. Pressure and flow were measured at $5 \mathrm{~cm}$ intervals along the tubes. Ultrasound flow probes (Transonic System Inc, Itheca, NY, USA) were used to record flow rate and a

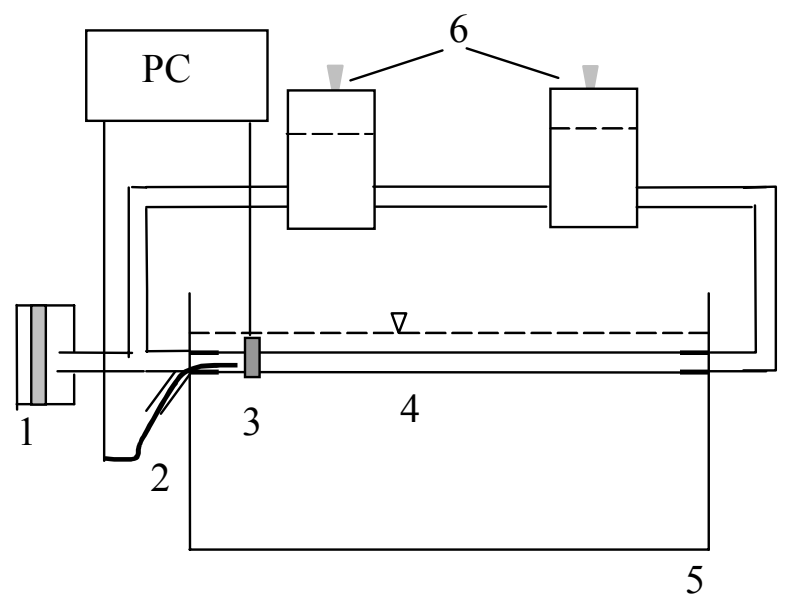

Fig.1. Setup of experiment. 1: Pump (Pulse generator); 2: Pressure transducer tipped catheters; 3: Flow probe; 4: Latex tubes of $200 \mathrm{~cm}$ in length; 5: Tank that contains water to a level just above the tube. 6: Water reservoirs.

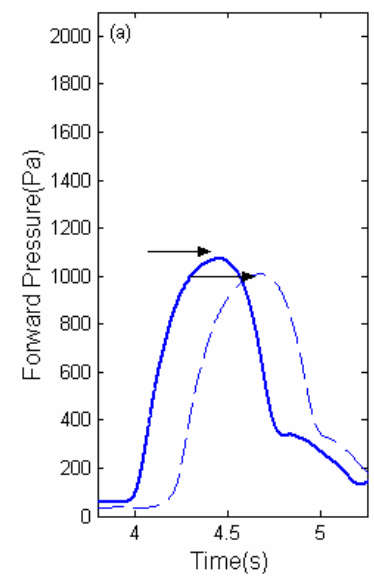

(a)

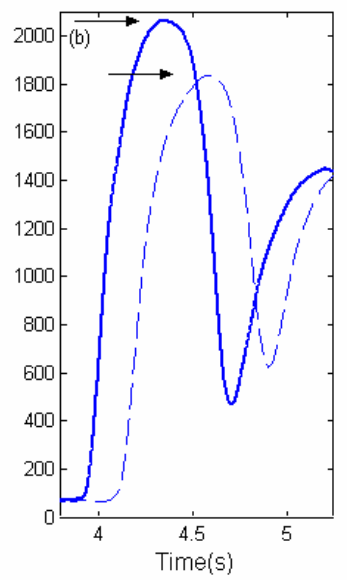

(b)
Fig. 2 Forward pressure wave in $16 \mathrm{~mm}$ normal tube (a) and modified tube (b). Solid line represents measurement of $20 \mathrm{~cm}$ and dash line represents $45 \mathrm{~cm}$ away from the inlet. Horizontal axis is time and vertical axis is forward pressure. The arrows indicate the magnitude of the forward pressure, while the difference between two arrows shows the dissipation of forward pressure between measurement of $20 \mathrm{~cm}$ and $45 \mathrm{~cm}$.

strain gauge transducer tipped catheter (Gaeltec, Scotland, UK) was used to measure the pressure. A total of 38 sets of pressure and flow were recorded for each tube. All data were acquired using Labview (National Instruments, Austin, Texas, USA) with sampling rates of $500 \mathrm{~Hz}$. Data were analysed using in house programs written in Matlab (The Mathworks, Natick, MA, USA).

In order to investigate the effect of the mechanical properties of the wall of the tube on wave dissipation, we repeated the experiment but with modifying the properties of the wall of the $16 \mathrm{~mm}$ diameter tube; a thread of strong cotton wound with a pitch of approximately $30^{\circ}$ around the circumference of the tube in the longitudinal direction.

\section{B. Analysis}

The measurement equipment of both flow and pressure were calibrated in advance before each experiment. With the flow meter, 1 Volt $=2 \mathrm{l} / \mathrm{min}$ for $8 \mathrm{~mm}$ flow probe. For the 16 $\mathrm{mm}$ flow probe, $1 \mathrm{Volt}=10 \mathrm{l} / \mathrm{min}$. Velocity was obtained from measured flow and corresponding area of flow probe. The pressure was calibrated using water column method. The pressure was linear proportion to voltage and the relationship between pressure and voltage was, $P=19865$ Volt-556, in Pascal.

The separated forward pressure wave, forward wave intensity were calculated using equations (5), (6) and (7). The separated forward pressure at the measurement of $20 \mathrm{~cm}$ and $45 \mathrm{~cm}$ away from inlet were shown in the Fig.2. The degree of wave dissipation within these two measurement sites was indicated by the difference of peak of forward pressure. The forward wave intensity had two peaks, which is shown in Fig3. The first one represents the forward compression wave and the second represents the forward expansion wave. Wave energy was calculated by integrating 


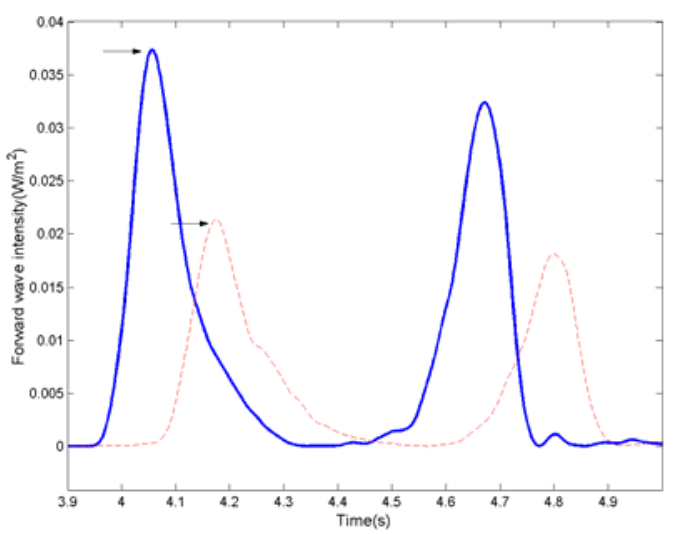

Fig.3. Forward wave intensity varied with time at measurement of $15 \mathrm{~cm}$ and $40 \mathrm{~cm}$ away from the inlet. Solid line represents measurement of $15 \mathrm{~cm}$ and dash line represents $40 \mathrm{~cm}$. Arrows indicate the maximum of wave intensity at measurement of $15 \mathrm{~cm}$ and $40 \mathrm{~cm}$, respectively.

the time history of wave intensity. The amplitude of forward pressure was obtained from the difference between first peak and static pressure and the values are presented in the Fig.4.

\section{RESULT}

\section{A. Dissipation of the forward pressure}

Fig.2 shows the variation of the forward pressure with time at known sites of measurement, where (a) indicates waveform of forward pressure for $16 \mathrm{~mm}$ normal tube and (b) for $16 \mathrm{~mm}$ modified tube. The peak of the forward pressure decreased exponentially with distance, which can be seen in Fig.4. At the same sites of measurement, the amplitude of forward pressure is highest in $8 \mathrm{~mm}$ diameter tube, the middle is $16 \mathrm{~mm}$ modified tube and the lowest is $16 \mathrm{~mm}$ normal tube.

\section{B. Dissipation of wave intensity}

The separated forward wave intensity are given in the Fig.3, where wave intensity varied with time and space is presented. The solid line is for $16 \mathrm{~mm}$ normal tube at measurement of $15 \mathrm{~cm}$ and dotted line is for $40 \mathrm{~cm}$. The arrows indicate the maximum of wave intensity at different sites, respectively, while the difference of two arrows indicates that the wave intensity dissipated when the wave was running from site of $15 \mathrm{~cm}$ to $40 \mathrm{~cm}$. Fig. 5 shows dissipation of the maximum of wave intensity with the travelled distance. Style of lines was used to specify the regression for experiments results in different sizes and properties of tube. The leading curve is for $8 \mathrm{~mm}$ diameter tube; the lowest is for $16 \mathrm{~mm}$ diameter normal tube and the middle one for $16 \mathrm{~mm}$ diameter modified tube.

\section{Dissipation of the wave energy}

Fig. 3 also shows the dissipation of wave energy. The area under the two peaks (compression wave and expansion wave) signifies the wave energy, while variation between the area under the solid peaks and dotted peaks implies wave energy dissipation during this phase. The dissipation of the

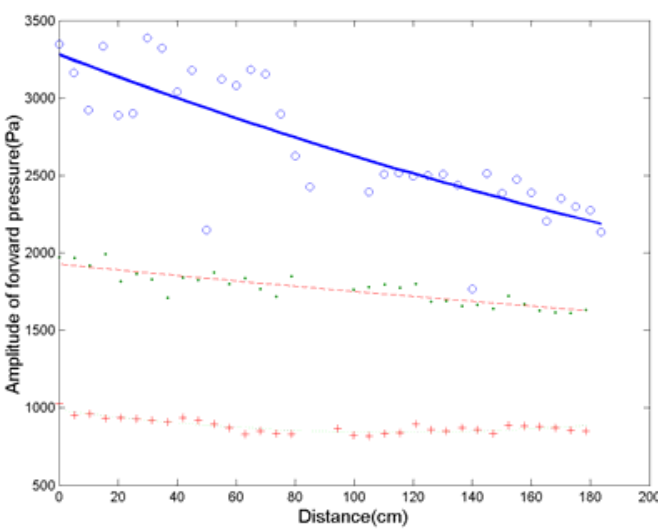

Fig.4. Amplitude of Forward pressure against distance travelled. Solid line (top) represents $8 \mathrm{~mm}$ tube, dash line (middle) represents $16 \mathrm{~mm}$ with modified wall and dot line (bottom) represents $16 \mathrm{~mm}$ normal latex tube. Horizontal axis is distance and vertical axis is amplitude of forward pressure. Symbol: 'o' is data for $8 \mathrm{~mm}$ tube, '.' for $16 \mathrm{~mm}$ modified tube and ' + ' for $16 \mathrm{~mm}$ normal tube.

wave energy against the distance is also shown in the Fig.6. One can see that the wave energy decreased exponentially along the distance travelled. Likewise, styles of lines were specified for three experiment results, respectively.

\section{DISCUSSION}

The experimental results show that the forward pressure decreased along the distance. In the $16 \mathrm{~mm}$ normal tube forward pressure decreased approximately $100 \mathrm{~Pa}$ between $20 \mathrm{~cm}$ and $45 \mathrm{~cm}$ away from the inlet, which is shown in the Fig.2. (a). This event can also be seen in Fig.4, where the amplitude of forward pressure is noticeably decreased with the distance under the three conditions. In addition, the effect of the size of the tubes and mechanical properties on the degree of dissipation of the forward pressure wave can be found in this Fig. One can see that within $200 \mathrm{~cm}$ the magnitude of forward pressure decreased approximately $40 \%$ in $8 \mathrm{~mm}$ diameter tube and $20 \%$ in the $16 \mathrm{~mm}$ diameter normal and modified tubes. It is clear that small sized tubes result in greater dissipation than large tubes do. For the same sized tubes, different mechanical properties have no discernable effect on wave dissipation.

The results in this study about the effect of size of tube on wave dissipation disagree with the previous findings by Anliker [4], in which the wave dissipation was not sensitive to the thickness ratio (wall thickness/tube radius)of tube. In our experiment, the wall thickness for both $8 \mathrm{~mm}$ tube and $16 \mathrm{~mm}$ tube were approximately $2 \mathrm{~mm}$. It is evident in our study that thickness ratio had an effect on wave dissipation. We believe that this difference in results might be attributed to the fact that the length of tube in our study was longer than that in other investigators' experiments.

Likewise, both wave intensity and wave energy dissipated exponentially with the distance, as seen in Fig.5 and Fig.6. More details about the dissipation of wave intensity and wave energy can be seen in the Fig.3. Maximum of wave intensity decreased approximately $40 \%$ between 


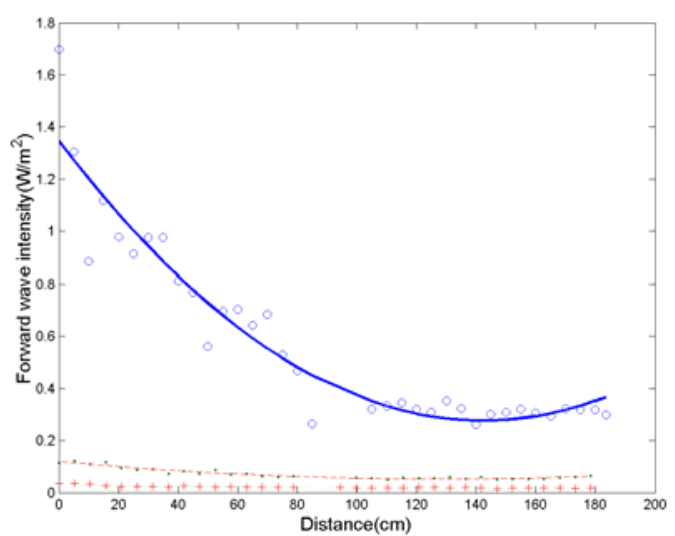

Fig.5. Maximum of wave intensity against distance travelled. Solid line (top) is for $8 \mathrm{~mm}$ tube, dash line (middle) for $16 \mathrm{~mm}$ modified tube and dot line (bottom) for $16 \mathrm{~mm}$ normal tube. Horizontal axis is distance. Vertical axis is maximum value of wave intensity. Symbol: 'o' is data for $8 \mathrm{~mm}$ tube, '.' for $16 \mathrm{~mm}$ modified tube and '+' for $16 \mathrm{~mm}$ normal tube.

measurement sites of $15 \mathrm{~cm}$ and $40 \mathrm{~cm}$. The area under two peaks of the solid line is clearly larger than that under the dash line.

Furthermore, the effect of both size of tube and mechanical property on wave intensity and wave energy dissipation are also shown Fig5 and Fig6. It can be seen in $8 \mathrm{~mm}$ tube that dissipation of maximum of wave intensity and wave energy was approximately $75 \%$ within $200 \mathrm{~cm}$ of distance; while in $16 \mathrm{~mm}$ tube it was approximately $50 \%$. It is evident that the size of tube has a strong effect on wave intensity and wave energy dissipation. In addition, the effect of mechanical properties on wave dissipation is also shown in those diagrams. The dissipation of wave intensity and wave energy in $16 \mathrm{~mm}$ modified tube was roughly equal to those in normal tube. This result gives further evidence that size of tube has a discernable effect on wave dissipation but the effect of mechanical property is not significant.

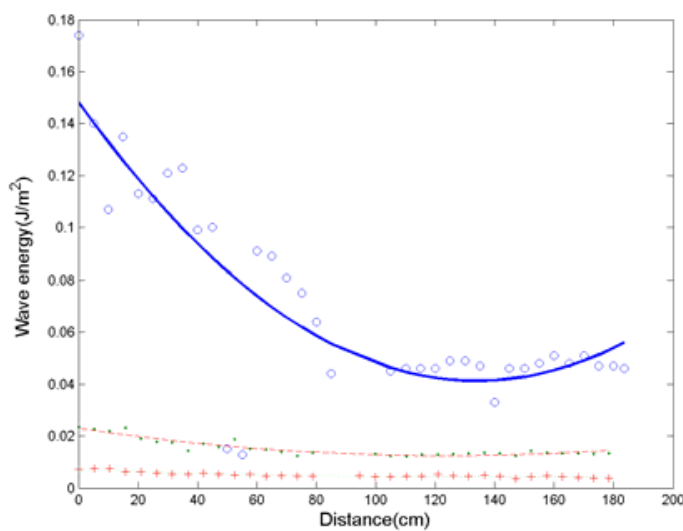

Fig.6. Forward wave energy against distance travelled. Solid line (top) represents $8 \mathrm{~mm}$ tube, dash line (middle) for $16 \mathrm{~mm}$ modified tube and dot line (bottom) for $16 \mathrm{~mm}$ normal tube. Horizontal axis is distance. Vertical axis is wave energy. Symbol: 'o' is data for $8 \mathrm{~mm}$ tube, '.' for $16 \mathrm{~mm}$ modified tube and '+' for $16 \mathrm{~mm}$ normal tube.

\section{CONCLUSION}

We conclude that the magnitude of the separated forward pressure, wave intensity and wave energy dissipated exponentially with the distance. The size of the tube has a significant effect on wave dissipation and the smaller the size of the tube, the greater the dissipation. The effect of the mechanical properties of the wall of the tube on wave dissipation is not discernable.

\section{REFERENCE}

[1] M Anliker, J.C. Stettler, and P. Niederer, Nonlinearity mathematical models of the arterial system: Effect of bifurcations, wall viscoelasticity, stenosis, and counterpulsation on pressure and flow pulse, Chapter 17, Handbook of Bioengineering.

[2] M Anliker, and J.A. Maxwell, "Dissipation and dispersion of small waves in arteries and veins with viscoelastic wall properties", Biophysical Journal, Vol. 8(8), pp.920-950, 1968.

[3] M. Anliker, M.B. Histand, and E. Ogden "Dissipation and attenuation of small artificial pressure waves in the canine aorta", Circulation Research, Vol. 23 (4), pp.539-551, 1968.

[4] M Anliker, G. William and E. Ogden, "Transmission of small pressure waves in the canine vena cava", American Journal of Physiology, Vol. 22(12), pp.644-651, 1971

[5] M. Anliker, "Wave Transmission Characteristics And Anisotropy of Canine Carotid Arteries", J. Biomechanics, Vol. 7, pp.151-154, 1974.

[6] D.L. Newman, S.E. Greenwald and H.T. Denyer, "Impulse propagation in normal and stenosed vessels", Cardiovascular Research, Vol. 15, pp.190-195, 1981

[7] C.D. Bertram, B.S. Gow and S.E. Greenwald "Comparison of different methods for the determination of the true propagation coefficient, in rubber tubes and the canine thoracic aorta", Med. Eng. Phys, Vol. 19 (3), pp.212-222, 1997.

[8] M. Ursino, E. Artioli and M. Gallerrani, “An experimental comparison of different methods of measuring wave propagation in viscoelastic tubes", J. Biomechanics Vol. 27(7), pp. 979-990, 1994

[9] K H Parker, and C.J.H. Jones, "Forward and Backward Running Waves in the Arteries: Analysis Using the method of Characteristics", Journal of biomechanical Engineering, Vol. 112, pp.322-326, August 1990

[10] K.H. Parker, J. Christopher, J. Dawson, and D. Gibson, “ What stops the flow of blood from the heart?", Heart Vessels, Vol. 4, pp.241-245, 1988.

[11] A.W.Khir, A. O'Brien, J. Gibbs, KH. Parker, "Determination of wave speed and wave separation in the arteries", J. Biomechanics, Vol. 34, pp.1145-1155, 2001.

[12] A.W. Khir, K.H. Parker "Measurement of wave speed and reflected waves in elastic tubes and bifurcation", J. Biomechanics, Vol. 35, pp.775-783, 2002 